\title{
Multilevel Reconfiguration Strategy for the System of Systems Engineering: Application to Platoon of Vehicles *
}

\author{
Pushpendra Kumar* Rochdi Merzouki* Blaise Conrard* \\ Belkacem Ould-Bouamama* \\ * LAGIS UMR CNRS 8219, Polytech Lille, University of Lille1 \\ 59655 Villeneuve d'Ascq, Lille, France (email: jahan.pushp@gmail.com; \\ rochdi.merzouki@polytech-lille.fr; blaise.conrard@polytech-lille.fr; \\ Belkacem.Ouldbouamama@polytech-lille.fr)
}

\begin{abstract}
In the present work, a generic method for multilevel modeling and reconfiguration of the System of Systems Engineering (SoSE) is proposed based on the bond graph modeling approach. The proposed formulation is applied to the road traffic dynamic for a platoon of Intelligent Autonomous Vehicles (IAVs). A bond graph model-based reconfiguration strategy is proposed for the multilevel model of the road traffic dynamic combining three levels namely submicroscopic, microscopic and macroscopic. This multilevel model is simulated for two scenarios of normal and faulty situations in a platoon of four IAVs.
\end{abstract}

Keywords: SoS, modeling, bond graph, reconfiguration, IAV.

\section{INTRODUCTION}

Large scale systems can be organized into various levels according to their complexity. The proliferation of new information and communication technologies (ICT) and hardware has increased the complexity of traditional systems which led to the introduction of the concept of System of Systems (SoS). This concept was introduced in $90 \mathrm{~s}$, and there are numerous definitions available for SoS (Jamshidi [2005]). Maier [1996] described the five characteristics of the SoS: operational independence, managerial independence, geographical distribution, emergent behavior, and evolutionary development.

In Defense Acquisition Guidebook [2010] SoS is defined as; a set of arrangement of independent systems that are related or connected to provide a given capability. The loss of any part of the system will degrade the performance or capabilities of the whole.

The concept SoS introduces the relationship between component systems (CSs) from an organizational point of view (Khalil et al. [2012]). The SoS has applications in many fields like engineering, management, biology, healthcare and defense. In the present work, we focus on the System of Systems Engineering (SoSE) and the modeling of such complex systems is challenging. Zhou et al. [2011] proposed agent-based modeling of the SoSE. Khalil et al. [2012] proposed hypergraph approach for modeling the SoSE. In the present work, we propose a method for modeling the SoSE based on power graphical modeling approach called bond graph (Paynter [1961]).

\footnotetext{
^ This work was supported by two European projects: Weastflows (196G) and InTraDE (091C), Interreg IVB north-west Europe (www.weastflows.eu and www.intrade-nwe.eu)
}

The fault in a CS may lead to failure of the global SoSE mission. Hence, it is important to detect and isolate the faults in CSs and based on this information reconfigure the SoSE for normal functioning. The causal and structural properties of the bond graph can be exploited for Fault Detection and Isolation (FDI) (Samantaray and OuldBouamama [2008]), and enable model-based reconfiguration (Pathak et al. [2008]; Loureiro et al. [2012]).

The concept of SoSE can be applied in the field of transportation as described in DeLaurentis [2005]. Hence, the proposed bond graph approach is applied to the road traffic dynamics for a platoon of IAVs. Such set of autonomous vehicles can describe an organization of the SoSE, because each elementary component system is operationally and managerially independant; they are dispatched geographically, with a continuous exchange of information; finally they can structurally make a selfreconfiguration of their organization.

Tampre and Arem [2001] described three levels in traffic dynamic namely submicroscopic, microscopic and macroscopic. Submicroscopic level describes the dynamics of an individual vehicle including its various components; microscopic level describes the dynamics of the interaction between the vehicles; and macroscopic level describes the whole traffic dynamics without distinguishing its component systems. Kumar et al. [2014] proposed a multilevel bond graph model of the road traffic dynamics combining sub-microscopic, microscopic and macroscopic levels. In the present work, a reconfiguration strategy is developed for this multilevel bond graph model.

The paper is organised in the following way: section 2 describes a generic method for the formulation of SoSE, in section 3 the proposed methodology is applied to a multilevel model of a platoon of vehicles, simulation results 
are discussed in section 4 for normal and faulty situations, finally the conclusion is given in section 5 .

\section{METHODOLOGY FOR MODELING THE SOSE}

Fig. 1 shows a generic multilevel representation of the SoSE. The SoSE is represented by various levels denoted by $L 1, L 2, L 3 \ldots L z$ and each level of the SoSE consists of CSs which horizontally interact (information exchange) with each other to achieve the goal of that level. Also, CSs interact vertically in different levels to achieve the global goal of the SoSE, combining all the levels. Any CS at a level is the subsystem of a CS at the higher level. For example, $C S_{n, L 1}^{i}$ represents the $n^{t h}$ subsystem in $L 1$ of the $i^{\text {th }}$ component system $C S_{i, L 2}$ in $L 2$. A multilevel model of the SoSE can be developed by combining all the levels. It helps to perform the CS fault detection and propagation from the lowest level to the highest level. The reconfiguration of the SoSE can be performed based on this multilevel model.

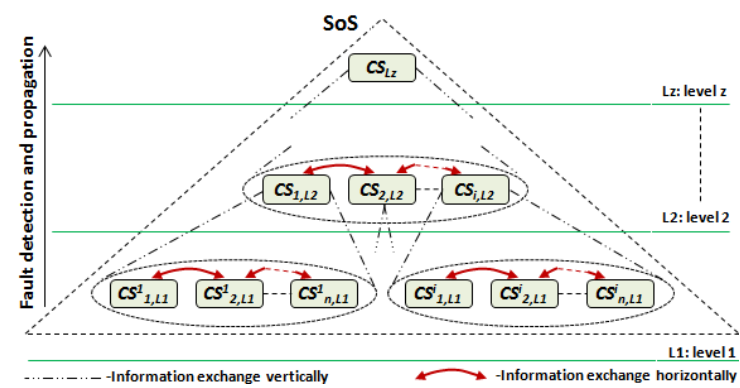

Fig. 1. Multilevel representation of the SoSE

The SoSE in Fig. 1 can be defined as $S_{o}=f\left(S_{m}, S_{c}, S_{r}\right)$, where $S_{o}$ represents the overall SoSE objective which depends on the SoSE model $S_{m}$, constraints $S_{c}$ and reconfiguration laws $S_{r}$ in case of faulty situations. $S_{m}$ is a set of dynamic mathematical relations to describe the horizontal and vertical interactions among the dynamic models of various CSs of the SoSE. $S_{c}$ is a set of constraints which must be satisfied every time if not then it represents the faulty situation and $S_{o}$ cannot be achieved. $S_{r}$ is a set of reconfiguration laws which recover the SoSE from faulty situations to normal situation for achieving $S_{o}$, may be with low performance.

\subsection{Modeling}

We develop dynamic model $\left(S_{m}\right)$ of the SoSE using energy based modeling approach called bond graph. The bond graph is a graphical modeling tool and can be denoted as $G(N, E)$, where $N$ is a set of nodes which represents the physical components/subsystems and called as junctions in bond graph, $E$ is a set of edges which represents the power bonds between nodes. These power bonds describe the power exchange between nodes based on two power variables namely effort $(e)$ and flow $(f)$. The physical CS are modeled using following elements: (i) active elements (sources of effort $S e$ and flow $S f$ ) which provide input power to the system, (ii) passive elements transform input power into dissipated (resistive element $R$ ) and stored (capacitance $C$ and inertia $I$ elements) energies (iii) and power conserving elements (effort conserve junction-0, flow conserve junction-1, transformer $T F$ and gyrator $G Y$ elements). The information exchange between the CSs can be modeled using activated bonds which represent only information exchange and no power transfer. The bond graph is very powerful to model in a unified way the physical systems of various natures and independently of the considered domain viz. mechanical, electrical, thermal etc. The bond graph approach allows understanding of the dynamic behavior of the process with a graphical vision and dynamic equations of the SoSE can be derived systematically from the bond graph. Moreover, the causal and structural properties of the bond graph can be exploited for the fault diagnosis and reconfiguration.

\subsection{Fault Diagnosis}

The bond graph modeling enables model based Fault Detection and Isolation (FDI). FDI method checks the consistencies between expected output and the actual output of a CS, which is described by the Analytical Redundancy Relations (ARRs). ARRs are the set of constraints $\left(S_{c}\right)$ among various parameters of a $\mathrm{CS}$, and can be deduced from the bond graph model by using structural properties of the bond graph. ARRs evaluation describes the difference between the expected and observed values, which is called as residual. The value of a residual defines a binary coherence vector whose elements are evaluated based on ARRs. The value of an element is ' 1 ' only if the corresponding residual is outside a predefined threshold, and it is ' 0 ' if residual is inside the predefined threshold. The coherence vector is calculated at every instant of time and a ' 1 ' value indicates a faulty situation. The faulty component is isolated based on a Fault Signature Matrix (FSM). FSM is a binary matrix which describes contribution of various components for each residual. A fault in a component is monitorable if it affects at least one residual, and a fault in a component can be isolated if its fault signature is different from the fault signature of all other components.

\subsection{Fault Accommodation through Reconfiguration}

The fault in any CS may lead to failure of the global mission of SoSE, so it is very important to recover the SoSE from the faulty situations by applying a robust reconfiguration strategy $\left(S_{r}\right)$. Once FDI procedure is applied on the SoSE, the faults can be monitored and a reconfiguration strategy can be proposed according to nature of the faults. The evaluation of the residuals enables to identify the fault in a CS at any instant of time and the SoSE recovers from the fault by reconfiguration at the same point of time. Thus, SoSE continues to function in normal as well as in faulty situations for achieving the global SoSE objective $S_{o}$.

\section{APPLICATION: A PLATOON OF VEHICLES}

In this section, the procedure described for modeling the SoSE in previous section is applied to a platoon of IAVs. A platoon of IAVs can be seen at different levels namely submicroscopic level, microscopic level and macroscopic level (Fig. 2).

Refere to Fig. 2, submicroscopic level $(L 1)$ represents the four considered $\operatorname{CSs}\left(C_{1, L 1}^{i}, C_{2, L 1}^{i}, C_{3, L 1}^{i}\right.$ and $\left.C_{4, L 1}^{i}\right)$ and 


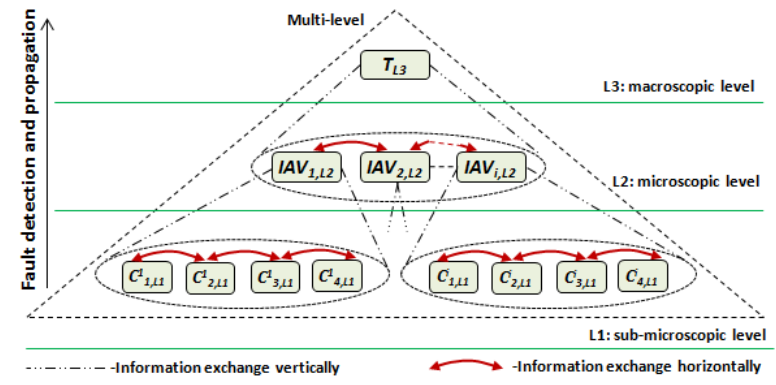

Fig. 2. Multilevel representation of a platoon of IAVs

their dynamic interaction for each $i^{\text {th }}$ IAV at microscopic level. These four CSs represent the dynamics of the four independently driven wheels of an IAV. Microscopic level $(L 2)$ represents the interaction between IAVs $\left(I A V_{i, L 2}\right)$ based on information exchange between them. Macroscopic level (L3) represents the dynamics of the whole traffic $\left(T_{L 3}\right)$ for a platoon of $i$ number of IAVs. Finally, all the three levels are combined to develop a multilevel model. This multilevel model is used for fault detection and to evaluate the effects of a faulty CS propagating from a lower level to the higher levels.

In the following subsections 3.1, 3.2, 3.3 and 3.4, the multilevel bond graph model for a platoon of IAVs is constructed in line with the model given by Kumar et al. [2014].

\subsection{Submicroscopic Level Modeling}

At submicroscopic level $L 1$, a two dimensional dynamic model of an IAV named RobuCar (Fig. 3(a)) is developed, the schematic representation of the considered IAV is shown in Fig. 3(b). The considered IAV has four traction wheels actuated by four independent direct current (dc) motors and all the wheels are steerable. IAV is equipped with an inertial sensor to measure its longitudinal, lateral, and yaw speeds. Also, sensors are mounted to measure the angular speed of each wheel and the current drawn by each motor. The following dynamics are considered for modeling: (i) traction actuators, slip and steering dynamics of the wheels and (ii) longitudinal, lateral and yaw dynamics of the vehicle's CM.

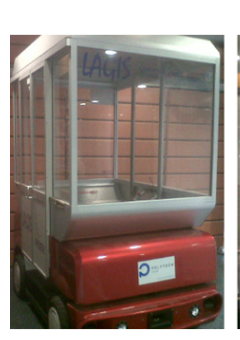

(a)
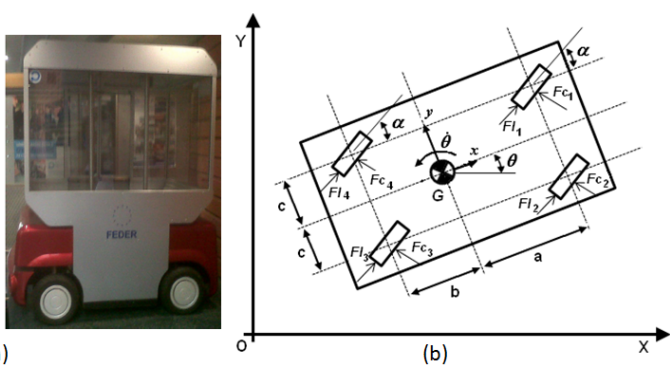

(b)
Fig. 3. (a) RobuCar at LAGIS (b) Schematic top view of an IAV

The complete bond graph model of an IAV considering all the dynamics is shown in Fig. 4. Fig. 4(a) shows the dynamics of vehicle's CM, where $m$ and $J$ represent mass and polar moment of inertia of the vehicle respectively. The dimensions of the vehicle are denoted by $a, b$ and $c$ in modulous of transformer elements. The full headed arrows corresponding to the speeds $(\dot{x}, \dot{y}, \dot{X}, \dot{Y}$ and $\dot{\theta})$ of the vehicle's $\mathrm{CM}$, represent the sensors to measure the value of the corresponding parameter.
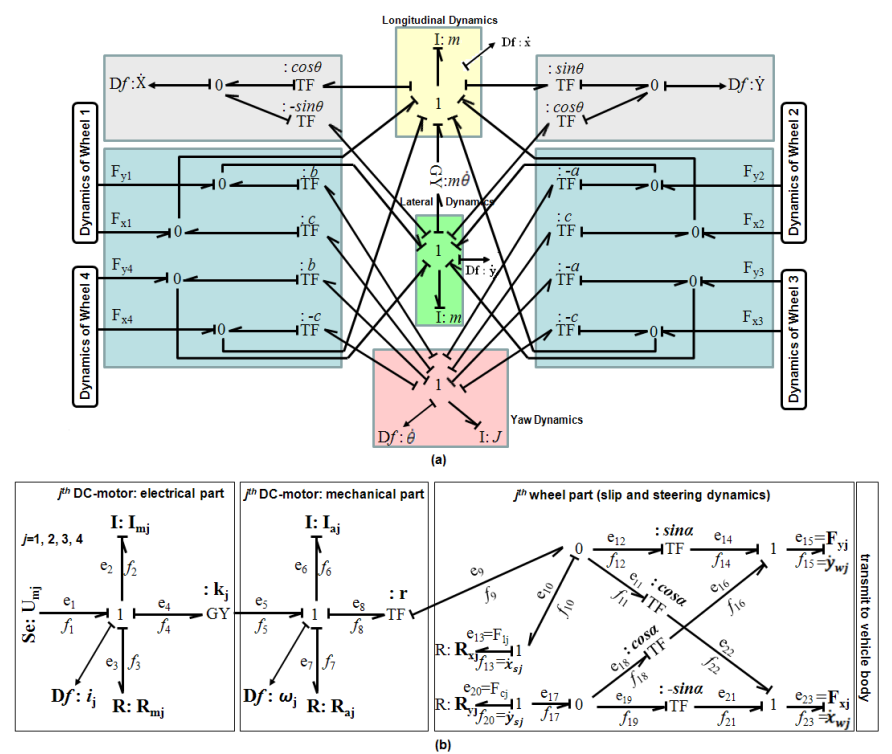

Fig. 4. Bond graph dynamic model of an IAV: (a) dynamics of the vehicle body (b) dynamics of the wheel

Any $i^{\text {th }}$ IAV is composed of four quarters of vehicle namely wheel-1 $\left(C_{1, L 1}^{i}\right)$, wheel-2 $\left(C_{2, L 1}^{i}\right)$, wheel-3 $\left(C_{3, L 1}^{i}\right)$ and wheel-4 $\left(C_{4, L 1}^{i}\right)$. Let's consider the dynamics of $j^{\text {th }}$ wheel- $j(j=1,2,3$ or 4$)$ as shown in Fig. 4(b). The wheel dynamics is composed of electric part of motor, mechanical part of motor, and slip and steering dynamics. In electrical part of the motor, $U_{m j}, I_{m j}, R_{m j}$ and $k_{j}$ represent voltage, inductance, resistance and torque constant of the motor respectively. In mechanical part of the motor, $I_{a j}$ and $R_{a j}$ represent polar moment of inertia and friction of the wheel-axle respectively. Angle $\alpha$ is the steering angle and $r$ is the radius of the wheel. $R_{x j}$ and $R_{y j}$ represent the slip contribution in $x$ and $y$ direction respectively, $e_{n}$ and $f_{n}$ represent the effort and flow respectively in $n^{t h}$ bond $(n=1,2,3 \ldots)$. The full headed arrows corresponding to current $i_{j}$ in motor and angular speed of the wheel axle $\omega_{j}$ represent the sensors to measure the value of the corresponding parameter.

The voltage source $(S e)$ provides voltage $U_{m j}$ to the motor. The electric power of the motor is converted into mechanical power with the help of gyrator element (GY). The longitudinal and lateral speeds of the wheel $\dot{x}_{w j}$ and $\dot{y}_{w j}$ respectively in conjunction with wheel's spinning speed generate the longitudinal and lateral slip speeds $\dot{x}_{s j}$ and $\dot{y}_{s j}$ respectively. The longitudinal force $F_{l j}$ and cornering force $F_{c j}$ are functions of the longitudinal and lateral slip speeds respectively. $F_{x j}$ and $F_{y j}$ are the forces generated by the wheel in $x$ and $y$ directions respectively, and are transmitted to the body of the vehicle in $x$ and $y$ directions respectively. The speed of the CM in inertial frame $X-Y$ is obtained by the transformation of $x-y$ frame by angle $\theta$. 


\subsection{Microscopic Level Modeling}

At microscopic level $L 2$, the interection between IAVs is such that the follower IAV always tries to maintain a safe seperation (interdistance) with leader IAV. For modeling this behavior between IAVs, they are connected by the spring-dashpot system. Actually, this physical connection is virtual, to represent the stick-slip motion of the follower IAV (Merzouki et al. [2013]).

In Fig. 5, a system of leader $\left(I A V_{n, L 2}\right)$ and follower $\left(I A V_{n+1, L 2}\right)$ IAVs is shown, where submicroscopic bond graph models of two IAVs are connected with the bond graph model of the spring-dashpot system, where bonds 2 and 1 connect the longitudinal dynamic junctions of the bond graph models of leader and follower IAVs respectively. The full headed arrow (bond 2) represents the flow activated bond, which tranfers only flow $f_{2}$ to the system and the effort $e_{2}$ is zero, this can be described as the motion of leader IAV is not influenced by the motion of follower IAV.

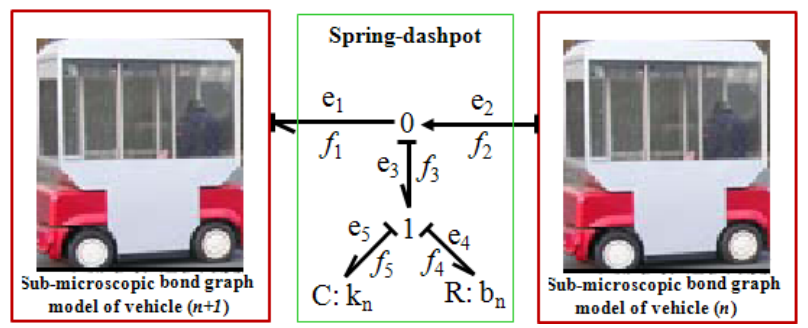

Fig. 5. Microscopic bond graph model showing interaction between two IAVs

\subsection{Macroscopic Level Modeling}

At macroscopic level $L 3$, the average values of macroscopic traffic variables (flow, density and mean speed)can be deduced from the bond graph model of microscopic level.

If $s_{n}$ is the distance headway for $n^{\text {th }}$ IAV which is given as $\left(X_{n}-X_{n+1}\right)$, and the state value of the position $X_{n}$ for each IAV is measured by the inertial sensor mounted on the vehicle, then average distance headway can be given by:

$$
\bar{s}=\frac{1}{i} \sum_{n=1}^{i} s_{n}
$$

Where $i$ represent number of vehicles at any instant of time in the considered road section. The average density $\rho$ of the traffic can be given in terms of average distance headway as following:

$$
\rho=\frac{1}{\bar{s}}
$$

If $\dot{X}_{n}$ is the speed of $n^{\text {th }} \mathrm{IAV}$, which is measured by the flow sensor mounted on the each vehicle, then average of $\dot{X}_{n}$ gives space mean speed $\bar{v}$.

$$
\bar{v}=\frac{1}{i} \sum_{n=1}^{i} \dot{X}_{n}
$$

Flow of the traffic $q$ can be calculated from the fundamental relation of the traffic flow:

$$
q=\rho \cdot \bar{v}
$$

\subsection{Multilevel Modeling}

A multilevel model of the road traffic dynamic for a platoon of IAVs is developed by combining all the three levels (Fig. 6).

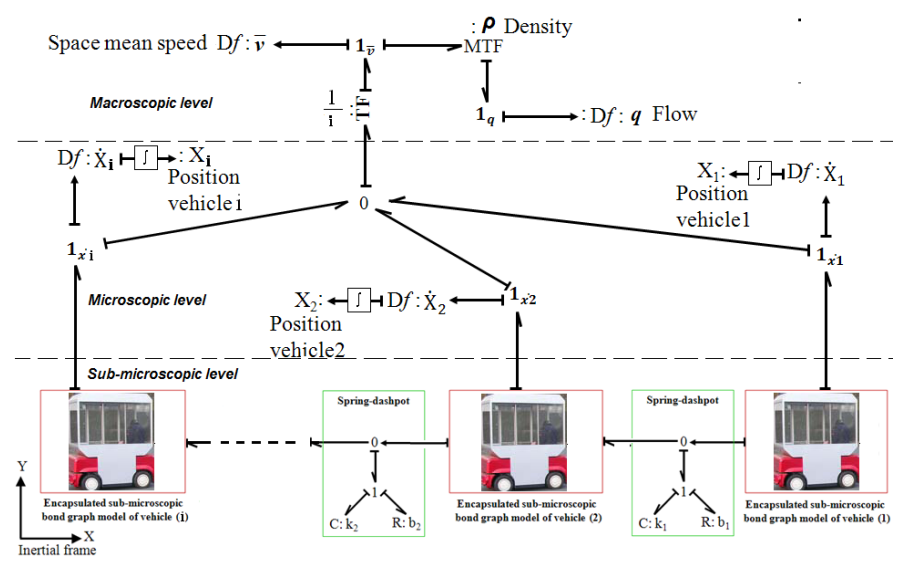

Fig. 6. A multilevel bond graph model for a platoon of IAVs

Refer to Fig. 6, the bond graph models of $i$-IAVs are connected by the bond graph models of virtual springdashpot systems. The junctions $1_{\dot{X}_{1}}, 1_{\dot{X}_{2}} \ldots 1_{\dot{X}_{i}}$ represent the speeds of IAVs $1,2 \ldots i$ respectively with respect to inertial frame, and the full headed arrows represent the sensors to measure the speeds $\dot{X}_{1}, \dot{X}_{2} \ldots \dot{X}_{i}$ of IAVs. The speeds of IAVs are integrated to get the positions $X_{1}$, $X_{2} \ldots X_{i}$ of each IAV. The space mean speed $\bar{v}$ and flow $q$ of the traffic are shown by the full headed arrows sensors, while the value of $\rho$ is calculated according to (1) and (2).

\subsection{Fault Detection and Isolation (FDI)}

In this section, we apply FDI method on the wheels of IAV for the fault identification. The structural and causal properties of the bond graph are used to generate ARRs for the wheels of any $i^{t h}$ IAV. ARRs are obtained directly from the bond graph by applying a well established methodology as described in Ould-Bouamama et al. [2003]. In the latter methodology, all the sensors are dualized into signal source of effort or flow ( $S S e$ or $S S f$ ), and the bond graph is assigned a preferred derivative causality. Then ARRs are derived from this bond graph by following the causal paths from known to unknown variables. Refer to Fig. 7, by applying the referred procedure two ARRs are derived (5) and (6) corresponding to the junctions where signal sources are connected.

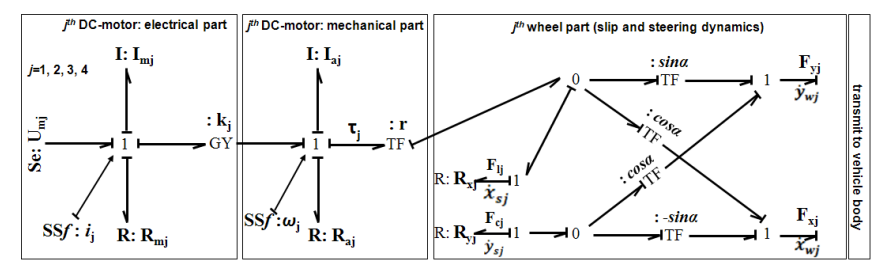

Fig. 7. Bond graph model of a wheel in preffered derivative causality

$$
A R R_{1 j}^{i}: U_{m j}-R_{m j} i_{j}-I_{m j} \frac{d i_{j}}{d t}-k_{j} \omega_{j}=0
$$




$$
A R R_{2 j}^{i}: k_{j} i_{j}-R_{a j} \omega_{j}-I_{a j} \frac{d \omega_{j}}{d t}-\tau_{j}=0
$$

$A R R_{1 j}^{i}$ and $A R R_{2 j}^{i}$ represent ARRs for the $j^{\text {th }}$ wheel of $i^{t h}$ IAV. These ARRs are evaluated by sensors data for FDI and if any of the two ARRs are found abnormal then there is a fault. For fault isolation, the ARRs are structurally analyzed to generate the FSM as shown in Table 1. In this analysis, the sensor faults are not considered and it is assumed that there is no sensor noise.

Table 1. FSM for the wheel system

\begin{tabular}{|l|l|l|l|l|}
\hline Components & $r_{1 j}^{i}$ & $r_{2 j}^{i}$ & $M_{b}$ & $I_{b}$ \\
\hline$U_{m j}$ & 1 & 0 & 1 & 0 \\
\hline$R_{m j}$ & 1 & 0 & 1 & 0 \\
\hline$D_{f}: i_{j}$ & 1 & 1 & 1 & 0 \\
\hline$I_{m j}$ & 1 & 0 & 1 & 0 \\
\hline$k_{j}$ & 1 & 1 & 1 & 0 \\
\hline$D_{f}: \omega_{j}$ & 1 & 1 & 1 & 0 \\
\hline$R_{a j}$ & 0 & 1 & 1 & 0 \\
\hline$I_{a j}$ & 0 & 1 & 1 & 0 \\
\hline$\tau_{j}$ & 0 & 1 & 1 & 0 \\
\hline
\end{tabular}

Refer to Table 1, the columns represent the components, residuals $\left(r_{1 j}^{i}\right.$ and $r_{2 j}^{i}$ are residuals for the $j^{t h}$ wheel of $i^{t h}$ IAV), fault monitorability $\left(M_{b}\right)$, and fault isolablility $\left(I_{b}\right)$. If a component is presented in an ARR then ' 1 ' is assigned in corresponding entry of FSM (means that the residual is sensitive to a fault in this component), otherwise ' 0 ' is assigned. It can be noticed from the Table 1 that none of the component has unique fault signature thus faults cannot be isolated but all faults are monitorable because each fault is sensitive to at least one residual.

\subsection{Reconfiguration}

We propose a reconfiguration strategy for a platoon of IAVs, which reconfigures the model in case of faulty situations based on the values of residuals at any instant of time. The residuals are calculated for the $j^{t h}$ wheel of an $i^{\text {th }}$ IAV in the platoon. Thus, the value of a residual at any point of time informs about the health of a wheel of an IAV in the platoon to which this residual is associated.

Refer to Fig. 8, a reconfiguration strategy is described for any $i^{\text {th }}$ IAV of the platoon. There are three cases for reconfiguration of the multilevel model based on four residual groups and each group represents the two residuals $r_{1 j}^{i}$ and $r_{2 j}^{i}$ of the $j^{t h}$ wheel of $i^{t h}$ IAV in the platoon.

Case I: If residuals $r_{1 j}^{i}$ and/or $r_{2 j}^{i}$ are/is triggered for a fault in any one wheel of the $I A V_{i, L 2}$ then the system can be reconfigured by making that wheel free and also making the wheel free at the opposite side of IAV corresponding to the faulty wheel. For example, if the front left wheel is faulty then make front left and front right wheels free. It avoids over steering of the vehicle because of angular speed difference between two opposite wheels. A wheel can be made free by removing the connection between the traction actuator and wheel; in that case the axle of wheel is not connected with motor and the wheel rotates freely. Mathematically, it can be achieved by assigning zero value to the torque constant $k_{j}^{i}$ for that wheel.

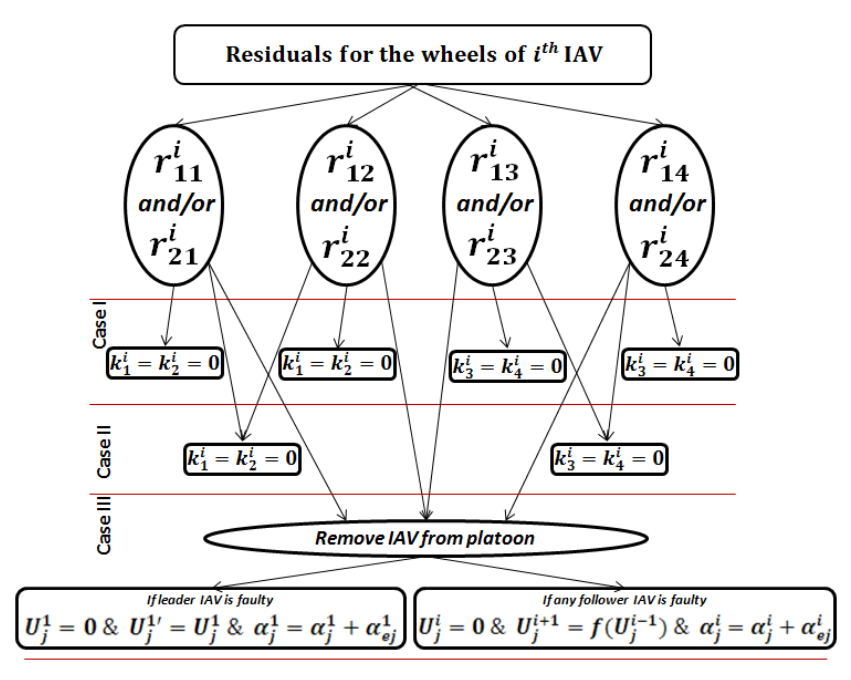

Fig. 8. Reconfiguration strategy

Case II: If two groups of residuals are faulty (only possible combinations are wheels-1,2 or wheels-3,4) then make those two wheels free by $k_{1}^{i}=k_{2}^{i}=0$ or $k_{3}^{i}=k_{4}^{i}=0$.

Case III: In any other case (say more than two wheels are faulty or two faulty wheels are not two opposite front/rear wheels), the faulty vehicle is removed from the platoon. If the faulty vehicle is any follower $i^{\text {th }} \mathrm{IAV}$, then $I A V_{i, L 2}$ is removed from the platoon by assigning zero value to its input voltage $U_{j}^{i}$ and give an extra steering angle (say $\alpha_{e j}^{i}$ ) to leave the platoon. Also, $I A V_{i+1, L 2}$ following the faulty $I A V_{i, L 2}$ is actuated with a voltage based on the interdistance model with $I A V_{i-1, L 2}$. Mathematically $U_{j}^{i+1}=f\left(U_{j}^{i-1}\right)$, which means that in the recofigured system, motion of $I A V_{i+1, L 2}$ depends on motion of $I A V_{i-1, L 2}$. If the faulty vehicle is leader $I A V_{1, L 2}$ then it is removed from the platoon in the same way $\left(U_{j}^{1}=0\right.$ and $\alpha_{j}^{1^{\prime}}=\alpha_{j}^{1}+$ $\alpha_{e j}^{1}$ ). But the follower $I A V_{2, L 2}$ becomes leader IAV in the reconfigured system and it is actuated by the input voltage $U_{j}^{1^{\prime}}$ equal to the voltage as given to the leader vehicle before fault.

\section{SIMULATION RESULTS}

The proposed multilevel model is simulated using a software called Symbols. SYMBOLS acronym stands for SYstem Modeling by BOndgraph Language and Simulation. It is a modeling, simulation and control systems software for a variety of scientific and engineering applications. For simulation, we consider a platoon of four IAVs and two scenarios are simulated for normal and faulty situations.

\subsection{First Scenario}

For the first scenario in simulation, we consider a platoon of four IAVs moving on a road without passing in normal situation. The simulation results are shown in Fig. 9. At submicroscopic level, Fig. 9(a) and Fig. 9(b) show the input voltage and the values of residuals for each wheel of the leader IAV (vehicle-1) respectively. it can be observe that the value of each residual is zero for vehicle1 , which represents the normal situation. At microscopic 
level, Fig. 9(c) and Fig. 9(d) show the $x$-direction position and speed of each vehicle with respect to time respectively. It can be observed that when a vehicle comes closer to its leader vehicle it decreases the speed and when seperation is more it increases the speed to maintain a safe interdistance which represents the stick-slip motion, the interdistance between the each pair of vehicles is shown in Fig. 9(c). At macroscopic level, Fig. 9(e) and Fig. 9(f) show the average values of density and flow of the traffic with respect to time respectively.
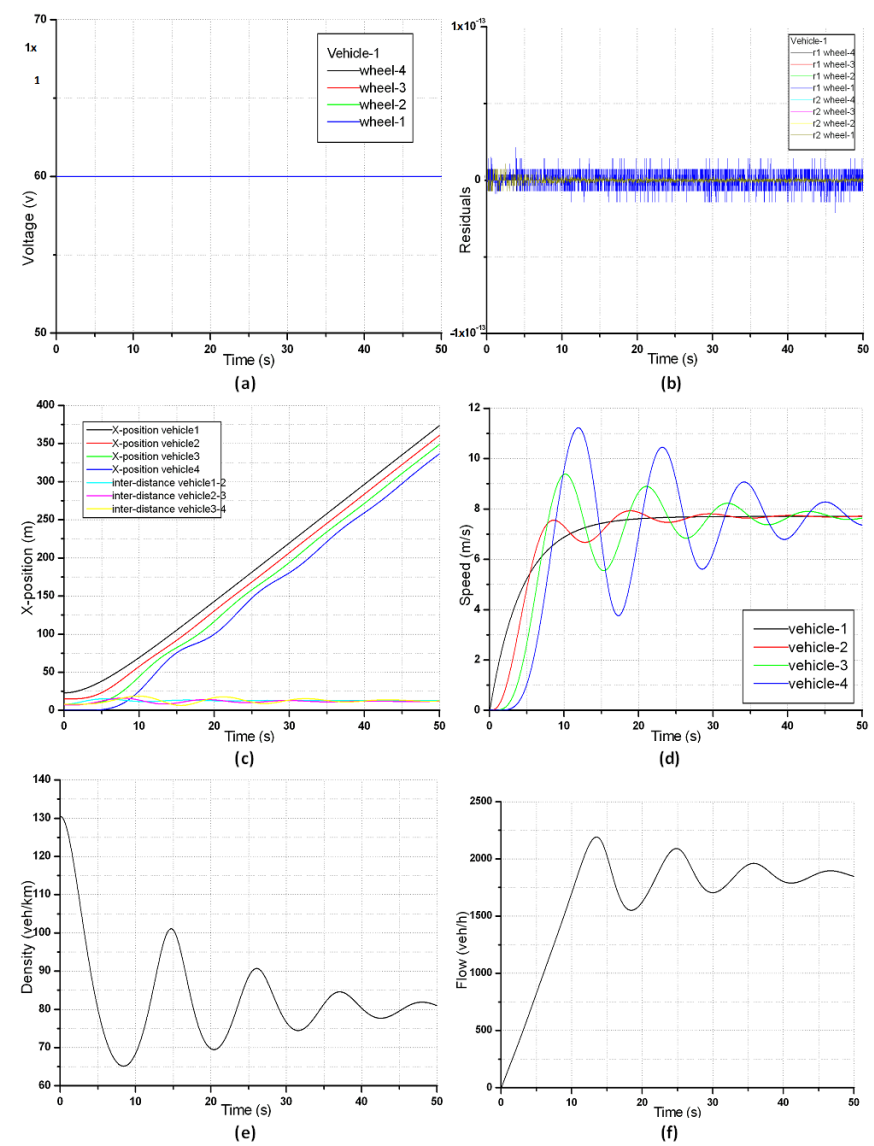

Fig. 9. First scenario in normal situation: (a) voltage in each wheel of leader vehicle (b) residuals for each wheel of leader vehicle (c) $x$-position of each vehicle and interdistance between vehicles $(d)$ speed of each vehicle (e) density of the traffic (f) flow of the traffic with respect to time.

\subsection{Second Scenario}

For the second scenario in simulation, we consider a platoon of four IAVs moving on a road without passing, and a fault is introduced in the front left wheel of the leader vehicle after $10 \mathrm{~s}$ by reducing its input voltage from $60 v$ to $25 v$ ( $58 \%$ reduction). The simulation results are shown in Fig. 10. Fig. 10(a) and Fig. 10(b) show the input voltage and the values of residuals for each wheel of the leader IAV (vehicle-1) respectively. It can be observed that the voltage in wheel-1 (front left) of vehicle-1 drops to $25 v$ after $10 s$ and at the same time corresponding residual $r_{11}^{1}$ is triggered to alarm about this fault. Fig. 10(c) and Fig. 10(d) show the $x$-direction position and speed of each vehicle with respect to time respectively. It can be observed that the speed of faulty leader vehicle decreases after $10 s$ and it leaves the platoon. Also, the interdistance between vehicle- 1 and vehicle- 2 becomes negative, because after occurring of the fault, vehicle- 1 moves in a curved path (Fig. 11(b)) due to the difference between the input voltages of the two front wheels.
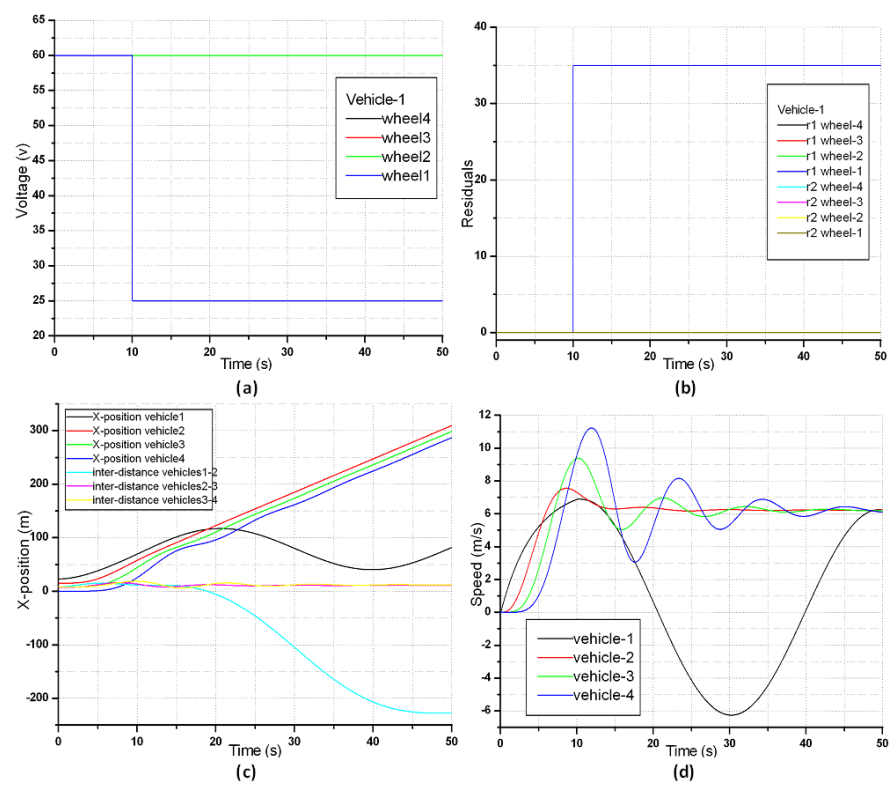

Fig. 10. Second scenario in faulty situation: (a) voltage in each wheel of leader vehicle (b) residuals for each wheel of leader vehicle (c) $x$-position of each vehicle and interdistance between vehicles(d) speed of each vehicle with respect to time.

Thus, this faulty situation leads to failure of the global mission of the SoSE and it becomes important to reconfigure it. Hence, the proposed reconfiguration strategy is applied to the model. The simulation results for the reconfigured model are shown in Fig. 11. Fig. 11(a), Fig. 11(b) and Fig. 11(c) show the trajectory of the leader vehicle in normal, faulty and reconfigured situations respectively. It can be observed that in the faulty situation, the leader vehicle moves in a curved path and leaves the platoon, but after applying the reconfiguration strategy (Case I in Fig. 8), it moves in the normal path. In Fig. $11(\mathrm{~d})$, the $x$-position of each vehicle is shown for the reconfigured system. From these results it can be concluded that after applying the proposed reconfiguration strategy to the model, it continues to fulfill the objective of the SoSE in faulty situation but with a low performance, because the final position of vehicle-1 is $375 \mathrm{~m}$ in normal situation (Fig. 9(c)) and it is $339 \mathrm{~m}$ in reconfigured model (Fig. 11(d)).

\subsection{Real-time Simulation}

The proposed multilevel model is simulated in realtime using a professional software dedicated for engineering and research applications called SCANeR ${ }^{\mathrm{TM}}$ (www.scanersimulation.com). It is a modular and realtime based structure. By using an Application Programming Interface (API), it is useful to integrate the emulated interdistance model between the vehicles. The management of the real-time traffic dynamic is done through the 


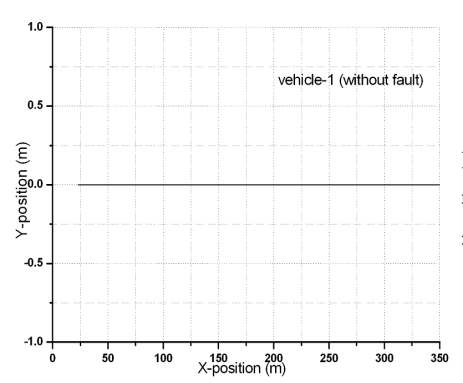

(a)
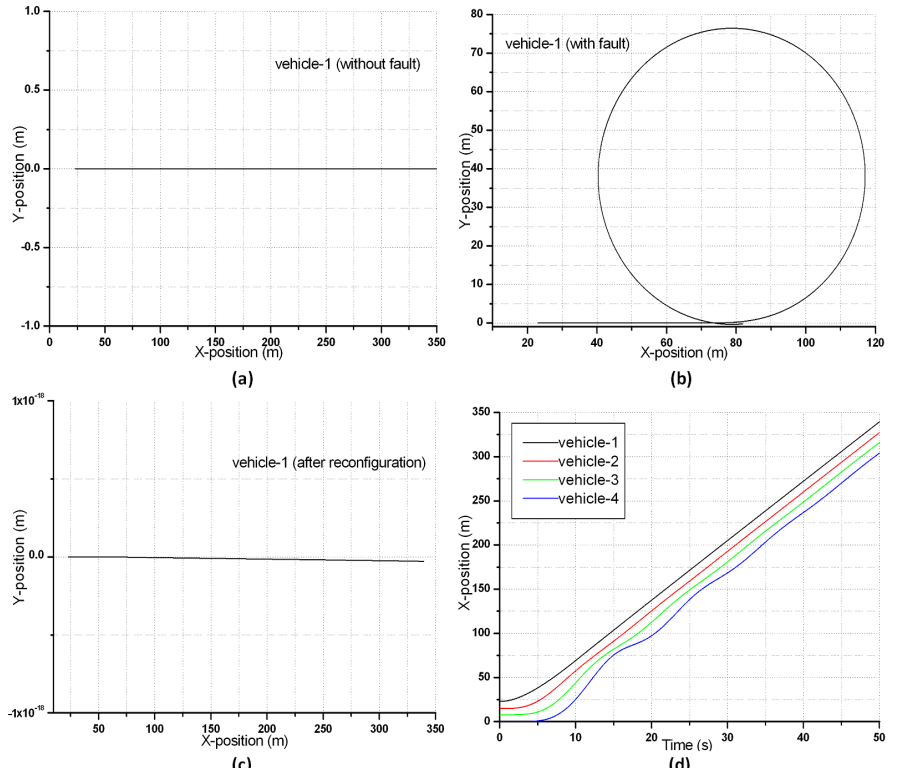

(b)

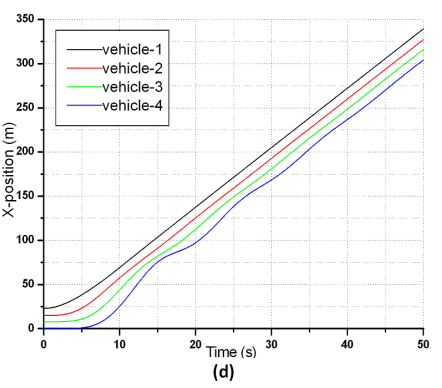

Fig. 11. Reconfiguration: (a) trajectory of the leader vehicle in normal situation (b) trajectory of the leader vehicle in faulty situation (c) trajectory of leader vehicle in after reconfiguration (d) $x$-position of each vehicle after reconfiguration

association of the 3D road mapping given in XML-format and the vehicle dynamics.

Refer to Fig. 12, the real-time simulation is performed for a platoon of four vehicles in normal situation, the follower vehicle follows the leader vehicle based on the interdistance model. The leader vehicle moves on a road for $55 \mathrm{~s}$ and then stops, the other follower vehicles follow it. Fig. 12(a) shows the $x$-direction position of each vehicle and the interdistance between the pair of vehicles. Fig. 12(b) shows the speed of each vehicle, it can be observed that the follower vehicles vary their speeds to maintain a safe interdistance, after $55 \mathrm{~s}$ the leader vehicle stops and the speeds of the follower vehicles also decrease to zero.
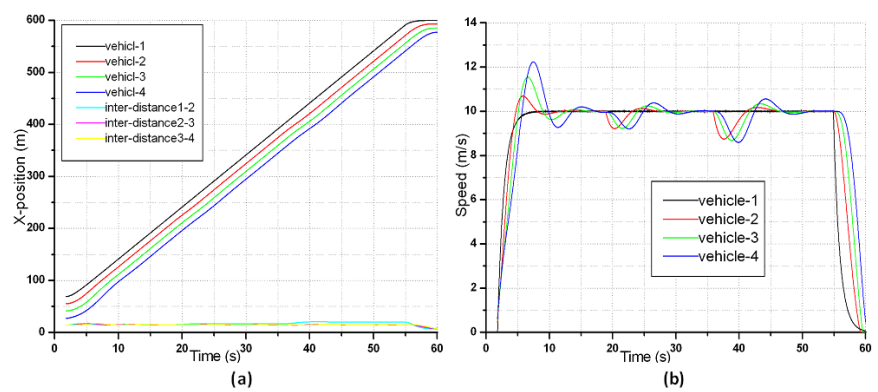

Fig. 12. Real-time simulation: (a) $x$-position of each vehicle and interdistance between vehicles (b) speed of each vehicle

\section{CONCLUSION}

The proposed bond graph approach for mofeling SoSE provides not only organizational model but also behavioral model by enabling physical modeling of the CSs, which is not the case in existing SoSE models. After analysing the simulation results for a platoon of four IAVs, it can be concluded that the multilevel model performs well in the normal situation as well as in the faulty situation after applying the proposed model-based reconfiguration strategy but with degraded performance in case of faults. In the future work, it is interesting to perform real-time simulations for more faulty scenarios.

\section{REFERENCES}

Maier., M.W. (1996). Architecting Principles for Systemsof-Systems. Proc. of the 6th Annual Symposium of INCOSE, pp 567-574.

Jamshidi, M. (2009). System of Systems Engineering: Principles and Applications. CRC Press, Taylor \& Francis Group.

Defense Acquisition Guidebook (DAG), Aug. 2010. Available online. http://at.dod.mil/docs/

DefenseAcquisitionGuidebook.pdf

Khalil, W., Merzouki, R., Ould-Bouamama, B., and Haffaf, H. (2012). Hypergraph Models for System of Systems Supervision Design. Systems, Man and Cybernetics, Part A: Systems and Humans, IEEE Transactions on, vol. 42, no. 4, pp. 10051012.

Zhou, B., Dvoryanchikova, A., Lobov, A., Minor, J., and Martinez Lastra, J. (2011). Application of the Generic Modelling Method for System of Systems to Manufacturing Domain. IEEE 37th Annual Conference on Industrial Electronics Society, pp. 352358.

Paynter, H.M. (1961). Analysis and Design of Engineering Systems. M.I.T Press, Cambridge, Massachusetts, USA.

Samantaray, A.K., and Ould-Bouamama, B. (2008). Model-based Process Supervision, A Bond Graph Approach. New York: Springer-Verlag.

Pathak, P.M., Samantaray, A.K., Merzouki, R., and OuldBouamama B. (2008). Reconfiguration of Directional Handling of an Autonomous Vehicle. IEEE Region 10 Colloquium and the Third ICIIS, Kharagpur, India.

Loureiro, R., Merzouki, R., and Ould-Bouamama, B. (2012). Bond Graph Model Based on Structural Diagnosability and Recoverability Analysis: Application to Intelligent Autonomous Vehicles. IEEE Transactions on Vehicular Technology, vol.61, no.3, pp.986-997.

DeLaurentis., D. (2005) Understanding Transportation as a System-of-Systems Design Problem. 43rd AIAA Aerospace Sciences Meeting, Reno, Nevada.

Tampre, C., and Arem, B.V. (2001) Traffic flow theory and its applications in automated vehicle control: a review. IEEE Intelligent Transportation Systems Conference Proceedings- Oakland (CA), USA, pp. 391-397.

Merzouki, R., Conrard, B., Kumar, P., and Coelen, V. (2013). Model based tracking control using Jerky behavior in platoon of vehicles. 12th European Control Conference, Zurich, Switzerland.

Kumar, P., Merzouki, R., Conrard, B., Coelen, V., and Ould Bouamama, B. (2014). Multilevel Modeling of the Traffic Dynamic. Intelligent Transportation Systems, IEEE Transactions on , vol.PP, no.99, pp. 1-17.

Ould-Bouamama, B., Samantaray, A.K., Staroswiecki, M., and Dauphin-Tanguy, G. (2003). Derivation of constraint relations from bond graph models for fault detection and isolation. In International Conference on Bond Graph Modeling and Simulation, Orlando, Florida. 